\title{
$\angle$ Research Square \\ Ox-LDL induced endothelial progenitor cells oxidative stress via p38/Keap1/Nrf2 pathway
}

\section{Qijun Jiang ( $\boldsymbol{\sim}$ jqjraymond@qq.com )}

Liyuan Hospital of Tongji Medical College of Huazhong University of Science and Technology https://orcid.org/0000-0002-9180-2396

\section{Chengpeng Li}

Liyuan Hospital of Tongji Medical College of Huazhong University of Science and Technology

\section{Zhigang Gong}

General Hospital of Central Theatre Command

\section{Zhigang Li}

General Hospital of Central Theatre Command

\section{Shifang Ding}

General Hospital of Central Theatre Command

\section{Research}

Keywords: Endothelial progenitor cells, Nrf2, ox-LDL, p38, Keap1, Apoptosis

Posted Date: April 2nd, 2021

DOl: https://doi.org/10.21203/rs.3.rs-348365/v1

License: (c) (i) This work is licensed under a Creative Commons Attribution 4.0 International License.

Read Full License

Version of Record: A version of this preprint was published at Stem Cells International on January 31st, 2022. See the published version at https://doi.org/10.1155/2022/5897194. 


\section{Abstract \\ Background}

In many studies, endothelial progenitor cells (EPCs) highly expressing antioxidant protein were induced oxidative stress and apoptosis by Oxidized-low density lipoprotein (ox-LDL). Nrf2 which was resently reported to regulate the antioxidant genes and cellular redox regulators was highly expressed in EPCs. However, its role in ox-LDL induced EPCs oxidative stress and apoptosis has not been fully illustrated.

\section{Methods}

EPCs isolated from human peripheral blood mononuclear cells were treated with different concentration of ox-LDL, Keap1 siRNA and a specific p38 MAPK inhibitor SB203580, then used to assay the whole cellular Nrf2 (total Nrf2, t-Nrf2), cytoplasmic Nrf2 (c-Nrf2), nuclear Nrf2 (n- Nrf2), NAD(P) H:quinone oxidoreductase 1 (NQ01) protein levels and Bax /Bcl-2 with western blot, NQ01 mRNA levels with RT-PCR, ROS level with H2DCF-DA, the loss/disruption of mitochondrial membrane potential (MMP) with JC-1, apoptosis with Annexin-V and PI,migration ability with transwell chambers and tube formation.

\section{Results}

The ox-LDL treatment decreased the n-Nrf2/Histone H3 to c-Nrf2/GAPDH ratio, NQ01 mRNA and protein expression levels. Treatment of ox-LDL enhanced the ROS production, induced loss of membrane potential, increase in cell shrinkage, pyknotic nuclei and apoptosis of EPCs. The Keap1 knockdown with Keap1 siRNA increased the nuclear translocation of Nrf2, the NQ01 mRNA and protein transcription levels, and prevented ox-LDL induced ROS generation and formation of JC-1 monomers. Treatment of oxLDL increased the activation of p38. Pretreatment with SB203580 significantly eliminated ox-LDL induced the inhibition of Nrf2 nuclear translocation, the depression of the mRNA transcription levels of NQO-1, the ROS generation and the formation of JC-1 monomers in EPCs. The pretreatment of Keap1 siRNA decreased the Bax/Bcl-2 ratio which was increased by the treatment of ox-LDL in EPCs. The oxLDL treatment decreased EPCs migration activity and tube formation. Whereas the pre-treatment with Keap1 siRNA preserved the migration ability and tube formation of EPCs

\section{Conclusion}

Ox-LDL induced EPCs oxidative stress and apoptosis via p38/Keap1/Nrf2 pathway.

\section{Background}

Occlusion of coronary artery and cerebral artery which is induced by atherosclerotic plaque formation and disruption is a major cause of mortality and morbidity within the societies living developed and modern 
lifestyles. Atherosclerotic plaque induced coronary artery and cerebral artery occlusion is one of the major causes of mortality and morbidity within the societies living developed and modern lifestyles.

Hypercholesterolemia-induced damage of vascular endothelium integrity is considered as an initial trigger of the development of atherosclerosis (1). Lipid oxidation, especially oxidized-low density lipoprotein (ox-LDL) plays a vital role in the development and progression of atherosclerosis. Ox-LDL induces atherosclerotic plaque formation primly through increased oxidative stress, endothelial cells damage, and impaired angiogenesis in ischemic tissue (2).

Endothelial progenitor cells (EPCs) considered to originate from hematopoietic stem cells play an important role in endothelial regeneration and vascular repair, by homing to sites of endothelial damage, differentiating into mature endothelial cells and incorporating into the endothelial lining. It was reported that antioxidant proteins were much more highly expressed in EPCs than in endothelial cells (3). However, recent studies showed that EPCs, unlike stem cells, are still vulnerable to environmental risk factors, particularly oxidative stress, which is abundant in the reparative ischemic environment (4).

Nuclear factor erythroid 2- related factor 2 (Nrf2) regulates the expression of genes encoding antioxidant proteins, and cellular redox regulators associated with human chronic diseases by binding to the antioxidant response element (ARE) (5). It plays an important role in the controls of redox balance, lipid metabolis, forming foam cells in atherosclerosis (6). Nrf2 is recently taken as a new potential pharmacological target for the treatment of many diseases, including cancer, neurodegenerative diseases, diabetes, airway disorders and cardiovascular disease (7). Nrf2 activity is regulated at multiple levels, including gene transcription, Kelch-like ECH-associated protein-1 (Keap1)-dependent and independent proteasome degradation, kinase-mediated phosphorylation, cytoplasm-nucleus trafficking, and DNA binding (8). Nevertheless, nuclear translocation is essential for Nrf2 and subsequent antioxidant regulation (9). Under homeostatic conditions, Nrf2 is retained in the cytoplasm by Keap1 and maintained at a low level by Keap1-dependent ubiquitination and proteasomal degradation system. In some cell types, ox-LDL has been reported to regulate Nrf2 activation and the transcription of antioxidant and oxidative stress response genes (10). However, whether ox-LDL influenced activation of Nrf2 and its related signals in EPCs has not been fully illustrated. In this work, we investigated the effects of ox-LDL on the level of activated Nrf2 in nuclear fraction of EPCs and the biological functions of EPCs, including migration and angiogenesis, as well as its relevance in oxidative stress and cell senescence.

\section{Methods}

Preparation of human EPCs

The study was approved by the Institutional Review Board at General Hospital of Central Theater Command. EPCs were prepared as previously described (11). Peripheral Blood Mononuclear Cells were isolated from peripheral blood of healthy human volunteers. Immediately after isolation, total mononuclear cells (MNCs, $5^{\star} 10^{6}$ cells $/ \mathrm{mL}$ medium) were plated on culture dishes coated with human fibronectin (F0895; Sigma) and maintained in endothelial basal medium (EBM, CC-3162; Lonza, 
Switzerland) supplemented with EGM Single Quots, $10 \mathrm{ng} / \mathrm{mL}$ VEGF (orb178366; Biorbyt, Missouri, USA) and $20 \%$ fetal bovine serum. The medium was replaced every 3 days. Adherent cells on 8 days of culture were stained by acetylated LDL and were labeled with Dil-acLDL (L3484, Invitrogen, Carlsbad, California, USA) and fluorescein isothiocyanate (FITC)-labeled lectin from ulex europaeus lectin (L9006; SigmaAldrich; Merck KGaA). Double-positive cells for Dil-acLDL and FITC-labeled lectin were identified as EPCs, as reported previously.

Cytoplasmic protein and nuclear protein extraction

Cells grown to $80 \%$ confluency and subjected to various treatments were subsequently washed with icecold Phosphate buffer saline (PBS) and it was prepared for protein extraction. The cytoplasmic protein and nuclear protein of EPCs was extracted using Nuclear protein and cytoplasmic protein preparation kit (P1200; BeiJing Applygen Technologies Inc, Beijing, China) according to manufacturer's instructions. Cells grown on $10-\mathrm{cm}$ dish were gently scraped with $3 \mathrm{ml}$ ice-cold PBS and it were centrifuged at $600 \mathrm{~g}$ for $10 \mathrm{~min}$ at $4^{\circ} \mathrm{C}$. After carefully aspirating the supernatant, cells were resuspended with $200 \mu \mathrm{l}$ ice cold CEBA Mix and proteinase inhibitor and incubated for $15 \mathrm{~min}$ on ice to allow cells to swell, followed by adding $11 \mu \mathrm{l}$ ice cold CEB-B. After vigorously vortexing for $10 \mathrm{~s}$ and centrifuging at $16000 \mathrm{~g}$ for $5 \mathrm{~min}$ at $4^{\circ} \mathrm{C}$, the supernatant (cytoplasmic fraction) were carefully aspirated and stored aliquots at $-80^{\circ} \mathrm{C}$. The pellet were resuspended with $200 \mu \mathrm{l}$ ice-cold NEB Mix and proteinase inhibitor and vigorously vortex. After vortexing, the suspension was placed on ice for $40 \mathrm{~min}$ before centrifuging at $16,000 \mathrm{~g}$ for $15 \mathrm{~min}$ at $4^{\circ} \mathrm{C}$. The supernatants (nuclear extracts) were stored aliquots at $-80^{\circ} \mathrm{C}$. Protein concentration of the supernatants was determined by the colorimetric assay (Bradford).

Western blot analysis

The protein samples were resolved on $12 \%$ Sodium dodecyl sulfate polyacrylamide gel electrophoresis (SDS-PAGE) and electro-transferred onto a nitro-cellulose membrane (IPFL00010; Millipore). The membrane was blocked with $5 \%$ non-fat milk and incubated with primary antibodies at a dilution of 1:1000 for $12 \mathrm{~h}$ at $4{ }^{\circ} \mathrm{C}$. The membrance were washed in TBS/T (Tris-buffered saline containing $0.2 \%$ Tween 20) and exposed to HRP-conjugated anti-goat IgG or anti-mouse secondary antibody (1:5000) for $1 \mathrm{~h}$, respectively, and then visualized by enhanced chemiluminescence detection reagents. The signal intensity of blotting was normalized to the Western signal of the corresponding total protein. Relative intensities of protein bands were analyzed by Image-pro plus6. 0 (Media Cybernetics, Silver Spring, MD, USA). Antibodies used in this study were: anti-Nrf2 (Ab62352; Abcam), anti-Histone H3 (PAB33309; BIOSWAMP), anti- glyceraldehyde-3-phosphate dehydrogenase (GAPDH, FNab03345; Wuhan Fine Biotech Co., Ltd. ), anti-NAD(P)H: quinone oxidoreductase 1 (NQ01) (Ab80588; Abcam), anti-Bax (FNab00810; Wuhan Fine Biotech Co., Ltd), anti-Bcl-2 (658701; BioLegend), anti-Keap1 (Ab139729; Abcam), anti-p-p38 (4511T; Cell Signaling Technology), anti-p38 (622403; BioLegend), and anti-beta-actin (PAB36265; BIOSWAMP).

RT-PCR Analysis. 
The treated cells in each group were collected, and the total RNA was extracted using Trizol. qPCR was performed with a total reaction volume of $10 \mu$, including $10 \mathrm{ng}$ of $\mathrm{CDNA}, 0.25 \mu \mathrm{M}$ forward and reverse primers and $5 \mu$ SYBR-Green qPCR master mix. The amplified genes and the primers used were as follows: NQ01 forward, CGCAGACCTTGTGATA and reverse, TGGCAGCGTAAGTGTA and GAPDH forward, ACAACTTTGGTATCGTGGAAGG and reverse, GCCATCACGCCACAGTTTC. GAPDH was used as a loading control. The qPCR reaction conditions included initial denaturing at $94^{\circ} \mathrm{C}$ for $3 \mathrm{~min}, 30 \mathrm{sec}$ denaturing at $94^{\circ} \mathrm{C}, 30 \mathrm{sec}$ annealing at $59^{\circ} \mathrm{C}$ and $30 \mathrm{sec}$ extension at $72^{\circ} \mathrm{C}$ for 40 cycles, then a final incubation at $65^{\circ} \mathrm{C}$ for $5 \mathrm{~min}$. For each sample, the number of cycles required to generate a given threshold signal (Ct) was recorded. Gene mRNA expression levels were analyzed using the $2 \Delta \Delta \mathrm{Cq}$.

Measurement of Intracellular Reactive Oxygen Species (ROS)

Intracellular ROS generation was detected by a fluorescence probe 2',7'-Dichlorofluorescin diacetate DCFH-DA (D6883; Sigma, USA) as previously described (12). The EPCs were cultured on slides in 6- well plates followed by exposure to the indicated experimental conditions, and the cells were incubated with 5 $\mathrm{mM} \mathrm{DCFH} \mathrm{DA} \mathrm{for} 20$ mins at $37^{\circ} \mathrm{C}$ in the dark. The slides were washed three times with serum-free EGM-2 medium. Subsequently, the representative images of ROS generation were captured using a Leica DMIL LED Inverted fluorescence microscope. Then the EPCs were resuspended in serum-free EGM-2, and the average fluorescence intensity of ROS in the cells was measured by flow cytometry (CytoFLEX S, BECKMAN). Samples without DCFDA were used as negative controls.

Analysis of mitochondrial transmembrane potential

Mitochondrial membrane potential (MMP) assay was performed by 5,5',6,6'-Tetrachloro-1,1',3,3'-tetraethylimidacarbocyanine iodide (JC-1) aggregates that are formed as a function of inner mitochondrial membrane potential. JC-1 selectively entere mitochondria and reversibly changes its color from red to green when the membrane potential decreases. After treatment, the cells were incubated in a humidified incubator at $37^{\circ} \mathrm{C}$ with $10 \mu \mathrm{g} / \mathrm{mL} \mathrm{JC}-1$ (T4069; Sigmal, USA) for $15 \mathrm{~min}$. Then cells were washed with serum-free EGM-2 medium three times, the fluorescence was monitored with a Leica DMIL LED Inverted fluorescence microscope (Leica, Germany) and flow cytometer (CytoFLEX S, BECKMAN, USA). The $\mathrm{red} / \mathrm{green}$ fluorescence ratio from five random fields was analyzed by using the Image-pro plus6. 0 (Media Cybernetics, Silver Spring, MD, USA). The $\Delta \Psi \mathrm{m}$ in each group was calculated as the ratio of red to green fluorescence.

Apoptosis Assay

EPCs apoptosis was quantified using an Annexin V-FITC/ propidium iodide (PI) Apoptosis Kit (40302ES20; Yeasen, USA) following manu facturer's instructions. EPCs were seeded into 6-well plates (2 $\times 105$ / well) and cultured for 24 hrs before experimental treatments. After treatment, EPCs were harvested and washed 3 times with PBS and resuspended at a density of $5 \times 105$ cells $/ \mathrm{mL}$. After washing, the cells were suspended in $1 \times$ Binding buffer, and $100 \mu \mathrm{L}$ of cell suspension was mixed with $5 \mu \mathrm{L}$ of FITC and 5 
$\mu \mathrm{L}$ of PI. After incubation for 15 mins in the dark, cell apoptosis was measured using a flow cytometer (CytoFLEX S, BECKMAN, USA).

In addition, morphological changes of the apoptotic EPCs nuclei were quantified by 4',6-diamidino-2phenylindole (DAPI, D9542; Sigmal, USA) staining. After treatment, $100 \mathrm{ng} / \mathrm{ml}$ DAPI was added in the culture media and incubated in $37^{\circ} \mathrm{C}$ incubator for 20 mins. Viable cells displayed normal nuclear size and uniform fluorescence, whereas apoptotic cells showed condensed, fractured or distorted nuclei. DAPIstained pyknotic nuclei were manually counted as percentage of 100 cells in each well.

Migration assay of EPCs.

Cell migration was quantified by a transwell chemotaxis assay using a Boyden chamber. To determine cell migrative ability, $1 \times 105$ cells were plated on the top side of polycarbonate Transwell fifilter ( $8 \mu \mathrm{m}$ pore size, MCEP24H48; Millipore, Massachusetts, USA) in 24-well plates. EBM-2 medium containing $10 \%$ fetal bovine serum (FBS) was added to the lower chamber. Then the growth medium was replaced with fresh EGM with medium containing ox-LDL. After 24 hours, the non-migrative cells in the upper chambers were gently removed and cells on the bottom membrane surface were fifixed with $4 \%$ paraformal dehyde for 15 mins, followed by stained with $0.5 \%$ crystal violet solution (PAB180004; Bioswamp, Wuhan, China), and counted under a microscope (Leica DMIL LED, Leica Company, Germany). Each experiment was performed in triplicate, and the number of migrated cells was determined from 5 random 100x fields per membrane.

EPC tube formation assay

The tube formation assay was performed using Matrigel to investigate EPCs angiogenesis. Matrigel (356237; CORNING, Germany) was dissolved at 4C, added to 48-well plates at $100 \mathrm{~mL}$ per well, and incubated at $37 \mathrm{C}$ for 30 minutes. EPCs $\left(2 \times 10^{4}\right.$ cells per well $\left.)\right)$ were seeded onto the Matrigel and cultured at $37^{\circ} \mathrm{C}$ in the presence of $5 \% \mathrm{CO} 2$. After $4 \mathrm{hrs}$, capillary-like structures were imaged under the microscope at $\times 100$ magnifification. The average branch point numbers that indicated tube formation were compared using Image Pro Plus 6. 0 (Media Cybernetics, Silver Spring, MD, USA).

Statistical analysis

All data were expressed as mean \pm SEM. Statistical analysis between two groups was performed using unpaired Student's $t$ test and comparisons between multiple groups were made by one-way ANOVA. Probability values were considered significant at $p<0.05$.

\section{Results}

EPCs morphology and identification

PBMCs were used for the preparation of human EPCs. Cells began to adhere to the wall after $24 \mathrm{~h}$ culture. Colonies were observed at $48 \mathrm{~h}$, and lots of colonies appeared at 4 days. After 8-day culture of 
mononuclear cells, spindle-shaped or cobblestone-like adherent cells were observed. Most of the adherent cells uptook Dil-ac-LDL and were positively stained with UEA-I (Fig. 1).

Ox-LDL inhibited Nrf2 nuclear translocation

To investigate the effect of ox-LDL on Nrf2 nuclear translocation in EPCs, the whole cellular Nrf2 (total Nrf2, t-Nrf2), cytoplasmic Nrf2 (c-Nrf2) and nuclear Nrf2 ( $\mathrm{n}$ - Nrf2) in EPCs after treatment of ox-LDL were determined by Western blot. The nuclear levels of Nrf2 were decreased to 21 . $9 \%$ by 12 hours treatment with $20 \mu \mathrm{g} / \mathrm{ml}$ ox-LDL (Fig. 2A, B). Analysis of c-Nrf2 and $\mathrm{n}$ - Nrf2 could further ascertain the location of Nrf2 and thereby could identify the influence of ox-LDL on Nrf2 preservation and nuclear translocation. Comparison of the n-Nrf2/Histone $\mathrm{H} 3$ to c-Nrf2/GAPDH ratio among all experimental groups showed that $10 \mu \mathrm{g} / \mathrm{ml}$ and $20 \mu \mathrm{g} / \mathrm{ml}$ ox-LDL respectively caused an decrease in the ratio of nuclear level of Nrf2 by 66 . $1 \%$ and $71.9 \%$ as compared to control group respectively $(P<0.05$, Fig. $2 B)$, with similar levels of $t-N r f 2$. However, the mRNA levels of Nrf2 were not influenced by treatment of ox-LDL(not showen), indicating that ox-LDL deceased n-Nrf2 level probably by the augmentation of the lysine acetylation and ubiquitination levels of Nrf2. To assess if Keap1-dependent or -independent proteasome degradation of Nrf2 is implicated in ox-LDL induced Nrf2 down-regulation, knockdown of Keap1 by transfection of Keap 1 siRNA was performed. The protein levels of Keap 1 were efficiently depleted by about $70 \%$ after 24h Keap1-siRNA treatment as evidenced by Western blot assay (Fig. 2C, D). Compared to those in the oxLDL treated group, the nuclear translocation of Nrf2 was substantially increased in the Keap1 knockdown treatment group (Fig. 2E, F). These results suggested that ox-LDL might decrease n-Nrf2 by Keap1dependent Nrf2 degradation and inhibition of nuclear translocation.

Nrf2 mediated ox-LDL inhibited NQ01 expression in EPCs

NQ01 a typical direct target gene of Nrf2 exerts a protective role in alleviating EPCs damage (13). Thus, the NQ01 mRNA levels is dependented on Nrf2 activity. To further examine the effects of ox-LDL on Nrf2 targeted genes in EPCs, we characterized concentration- and time-dependent regulation of NQ01 mRNA by ox-LDL in EPCs through realtime RT-PCR. Results showed that after 10 $\mathrm{gg} / \mathrm{ml}$ ox-LDL treatment, transcript levels of the NQ01 gene sharply decreased to $67.9 \%$ at $6 \mathrm{~h}$, reached a minimum of $39.4 \%$ at $18 \mathrm{~h}$, and returned to control levels by $48 \mathrm{~h}$ and later (Fig. 3A). Consistently, ox-LDL treatment decreased NQ01 levels in a dose-dependent manner (Fig. 3B). NQ01 expression levels attained a minimal level at $20 \mu \mathrm{g} / \mathrm{ml}$ after $18 \mathrm{~h}$ treatment(Fig. 3B). Additionally, pretreatment of Keap1 siRNA transfection 24h before ox-LDL treatment resulted in a significant increase of NQ01 transcription compared to those treated by ox-LDL without Keap1 siRNA (Fig. 3C).

To determine whether ox-LDL influences NQ01 protein expression level, we performed immunoblotting analysis of NQ01 protein. In EPCs challenged with $20 \mu \mathrm{g} / \mathrm{ml}$ ox-LDL for $24 \mathrm{~h}$, the NQ01 protein level was markedly decreased, and pretreatment of Keap1 siRNA transfection for 24h significantly promoted NQ01 levels (Fig. 3D, E). These results indicated that ox-LDL reduced NQ01 expression at the transcriptional and protein level in a time- and concentration-dependent manner in EPCs, which might been implicated to Keap1-dependent regulation of Nrf2. 
Previous studies showed that redox in EPCs is regulated by several signal transduction pathways. The mitogen-activated protein kinase (MAPK) p38 recently identified as a modulator of ex vivo progenitor cells proliferation, plays a primordial role in response to changes in the cellular redox (14). MAPK p38 was reported to suppress Nrf2 activation via the promotion of stabilization of the interaction between Keap1 and Nrf2 and the breakdown of Nrf2 in various human cell lines (15). To elucidate the molecular mechanisms underlying the inhibition of Nrf2/NQ01 by ox-LDL, we evaluated the effect of ox-LDL on phosphorylation of the MAPK p38 in EPCs with Western blot. Results showed that 20ng/ml ox-LDL treatment for 2 hours significantly increased the activation of p38(Fig. 4A,B). To further understand the role of p38 MAPK in EPCs redox regulation, we investigated the impact of p38 MAPK on Nrf2 nuclear translocation and NQ01 expression in EPCs, useing a specific p38 MAPK inhibitor SB203580. Results showed that $1 \mu \mathrm{M}$ SB203580 pretreatment significantly eliminated ox-LDL induced inhibition of Nrf2 nuclear translocationand depression (Fig. 4C and D) and the mRNA expression levels of NQO-1 in EPCs (Fig. 4E). However, the mRNA and protein level of Keap1 has not been influenced by SB203580 (not showen). These results suggest that ox-LDL inhibited the Keap1/Nrf2 antioxidative defense pathway probably via activating p38 signaling pathway.

Nrf2 mediated ox-LDL induced EPCs oxidative stress and apoptosis

To determine the role of Nrf2 inhibition and p38 MAPK in ox-LDL induced oxidative stress in EPCs, the effects of Keap1 siRNA and SB203580 on ox-LDL induced ROS generation were assessed by detecting the oxidation of 2',7'-Dichlorofluorescin diacetate (H2DCF-DA) with fluorescent microscopy and flow cytometry. Treatment of ox-LDL for 6 hours significantly enhanced ROS production in EPCs, measured by dihydroethidium (DHE) staining with fluorescent microscopy. However, the pretreatment with Keap1 siRNA and SB203580 both significantly prevented ox-LDL induced ROS generation in EPCs (Fig. 5A, B). Similar results were obtained using flow cytometry as analyser. Keap1 siRNA and SB203580 both significantly reduced EPCs ROS level which was elevated by ox-LDL (reduced from 34. 99\% to 17. 40\% and $21.42 \%$ respectively $P<0$. 0001), and SB203580 completely blocked elevated ROS in the presence of Keap1 siRNA. These results indicated that the increase of ROS production in EPCs after ox-LDL stimulation primarily attributed to the inactivation of Nrf2 and activation of p38.

One of the early critical events in apoptosis is the loss/disruption of MMP, which eventually causes the initiation and activation of apoptotic cascades (16). The effects of ox-LDL on EPCs mitochondrial transmembrane potential was evaluated with $\mathrm{JC}-1$ using fluorescence microscope and flow cytometry. Treatment of $20 \mu \mathrm{g} / \mathrm{ml}$ ox-LDL significantly increased the formation of monomeric JC-1 with green fluorescence in mitochondria, indicative of a loss of membrane potential (Fig. 6A). Keap1 siRNA and SB203580 pretreatment decreased the formation of JC-1 monomers (Fig. 6B), suggesting that activation of Nrf2 and inhibition of p38 protect EPCs from apoptosis.

The effects of ox-LDL on survival of ex vivo expanded EPCs was then examined with both manually counting DAPI stained pyknotic nuclei and flow cytometric analysis of Annexin-V and PI. The Annexin- 
V/PI double staining showed that treatment of $20 \mu \mathrm{g} / \mathrm{ml}$ ox-LDL for $6 \mathrm{~h}$ increased the apoptotic proportion of EPCs from $9.13 \%$ to $44.30 \%$ (Fig. 7A), concomitant with an increase in cell shrinkage and pyknotic nuclei (Fig. 7B, C). However, pretreatment with SB203580 decreased ox-LDL induced EPCs apoptotic proportion to $22.03 \%$ (Fig. 7A, B and C).

To determine whether the apoptotic pathway was implicated to ox-LDL induced apoptosis, the expression level of the pro-apoptosis factors, Bax and the pro-survival factor, $\mathrm{Bcl}-2$, were examined with Western blot. As shown in Fig. 8A and B, ox-LDL increased 2 folds of the expression of Bax, and simultaneously decreased $30 \%$ of the expression of $\mathrm{Bcl}-2$, eventually leading to an approximate 4 -folds of Bax/Bcl-2 ratio increase in EPCs, whereas these effects were respectively blocked by the pretreatment of Keap1 siRNA transfection or SB203580. All these results suggested that activation of Nrf2 and inhibition of p38 significantly inhibited ox-LDL induced ROS production, mitochondria membrane potential reduction and cell apoptosis, and activated apoptotic pathway as increases of $\mathrm{Bax} / \mathrm{Bcl}-2$ ratio.

Ox-LDL inhibited EPCs migration and tube formation via Nrf2

The migration activity of EPCs 2 hours after ox-LDL stimulation was detected using transwell chamber assay. The transwell chambers were incubated in a $37^{\circ} \mathrm{C}$ incubator for 24 hours. The migrated EPCs were stained with $1 \%$ crystal violet solution and counted in five random high-power (100x) microscope fields. Results showed that treatment with ox-LDL significantly reduced the migration ability of EPCs (Fig. 9A). To test the role of Nrf2 signal in the loss of EPCs migration activity, Keap1 siRNA was pretreated $0.5 \mathrm{~h}$ before addition of ox-LDL to EPCs culture media. Results showed that Keap1 siRNA preserved the migration ability of EPCs (Fig. 9C).

The present study investigated the effect of ox-LDL and Keap1 siRNA on the angiogenesis of EPCs in vitro by performing a tube formation assay. The formation of tube-like structures was decreased in oxLDL treated EPCs, whereas pre-treatment with Keap1 siRNA significantly increased the tube formation of EPCs (Fig. 9B, D). The results suggested that activation of p38 attenuated ox-LDL induced EPC tube formation inhibition.

\section{Discussion}

Atherosclerosis which contributed to many vascular diseases, is thought to be initiated and promoted by oxidative stress and vascular endothelial injury (17). Endothelial cells usually work as the first defensive line in vessels against harmful substances in the blood. However, when vascular endothelial cells are exposed to excessive external stimulus, their structure and function become impaired (18). EPCs with the inherent capacity to differentiate into mature endothelial cells play a critical role in postischemic vascular repair (19). Ox-LDL which accumulates in the vascular wall promotes the formation and development of atherosclerotic plaques by inducing vascular endothelial apoptosis and impairment of EPCs function (20). It was reported that the antioxidant genes were much more higher expressed in EPCs than that in endothelial cells (21). However, in the present study, we found that ox-LDL increased EPCs ROS production, mitochondria dysfunction and apoptosis. The impaired EPCs function might influence the 
balance between damage and repair of endothelial cells and accelerate formation of atherosclerosis. These results were consistent with the previous reports in which ox-LDL exerted a deleterious effect on ability and survival of EPCs via ROS generation and antioxidant enzymes dysfunction.

Nrf2 is ubiquitously expressed in cells and is closely associated with AS pathogenesis (22). In endothelial cells, activation of Nrf2 was reported to ameliorate survival, proliferation, and angiogenic function of endothelial cells in vitro and in vivo (23). The activation of Nrf2 is now widely considered to be controlled by Keap1 which acts as a cysteine-based mammalian intracellular sensor for exogenous and endogenous electrophiles and oxidants (24). In the condition of oxidative or electrophilic stress, Nrf2 releases from Keap1 in the cytoplasm, translocates to the nucleus, binds to ARE and induces the transcription of many antioxidant genes, such as NQ01 (25). NQ01 as a cytosolic oxidoreductase plays a critical role in regulating the ROS levels. NQ01 catalyze the oxidation of Nicotinamide adenine dinucleotide phosphate $(\mathrm{NAD}(\mathrm{P}) \mathrm{H})$ to $\mathrm{NAD}(\mathrm{P})+$, and depressed the ROS levels through inhibition of quinones from entering the one electron reduction to semiquinone free radicals and ROS (26). So these powerful antioxidant protein eliminates accumulated ROS quickly and maintains cells redox balance. In the present study, we found that ox-LDL reduces the nuclear translocation of Nrf2 and also NQ01 expression at the transcriptional and protein level in EPCs in a time- and concentration-dependent manner. However, we also found that ox-LDL did not influence the mRNA expression levels of Nrf2, suggesting that ox-LDL probably inhibited Nrf2 not through inhibition of Nrf2 mRNA transcription. (A)

To further define the role of Keap1 in the regulation of Nrf2 activity, we employed Keap1 siRNA to regulate Nrf2 signal. The results showed that Keap1 siRNA increased of NQ01 mRNA and protein transcription levels. These results suggested that ox-LDL might inhibit Nrf2/ARE system through Keap1-dependent Nrf2 degradation and inhibition of nuclear translocation.

p38 MAPK signal pathway which is activated by many environmental factors and extracellular physiological stimuli, plays an important role in the pathogenesis of atherosclerosis (27). p38 MAPK signal was reported to induce endothelial cell inflammation and apoptosis (28). It has been reported that ox-LDL elicited increased p38 MAPK phosphorylation and tremendous ROS generation in HUVECs (29). Whereas previous studied showed that p38 MAPK inhibits Keap1/Nrf2/ARE pathway via the regulation of Nrf2 stability (30). Present study showed that ox-LDL significantly activated $p 38$. We also found that inhibition of p38 MAPK reversed ox-LDL induced inhibition of Nrf2 nuclear translocation and NQO-1 mRNA transcription in EPCs. These results suggest that ox-LDL inhibited the Keap1/Nrf2 antioxidant defense pathway probably via activating p38 signaling pathway. The present study also found that inhibition of p38 MAPK significantly reversed ox-LDL induced the ROS generation, mitochondrial dysfunction and apoptosis. The results suggest $\mathrm{p} 38 / \mathrm{Keap} 1 / \mathrm{Nrf2}$ pathway play a central role in control of EPCs oxidative stress and apoptosis.

Several studies showed that the migration and tube formation of EPCs were impaired in many pathological conditions (31). Oxidative stress has always been implicated in mediating the apoptosis and dysfunction of EPCs (32). In the present study, we also found that upregulation of Nrf2 through the Keap1 
knockdown decreased the EPCs ROS generation and mitochondrial dysfunction, and ameliorated the migration ability and tube formation of EPCs. These results reveal that oxidative stress play a central role in the connecting the Nrf2 activity to the EPCs function, and were consistent with previous reportes in which lack of Nrf2 attenuated survival, proliferation, and angiogenic function of EPCs (33).

Bax and Bcl-2 have been identified to be involved in the control or execution of apoptosis (34). Bax permeabilizes the outer membrane of mitochondria and succeeds the release of cytochrome $c$, a proapoptotic molecule. Cytosolic cytochrome $\mathrm{c}$ then could bind with many pro-apoptotic factors to create a protein complex known as an apoptosome. However, Bax effects could been inhibited by Bcl-2, so the bax/Bcl-2 ratios have been implicated in the intrinsic apoptotic pathway triggered by mitochondrial dysfunction (35). The present study showed that ox-LDL increased Bax expression and decreased Bcl-2 expression, resulting in a significant increases of $\mathrm{Bax} / \mathrm{Bcl}-2$ ratio within EPCs. These effects were partially blocked by the Keap1 knockdown or p38 inhibition. These results suggested that upregulation of Nrf2 signal inhibited the activation of apro-apototic signal pathway which was induced by ox-LDL.

\section{Conclusions}

All these results suggest that ox-LDL induced EPCs oxidative stress by Keap1-dependent Nrf2 degradation and inhibition of nuclear translocation. Treatment of ox-LDL inhibited the Keap1/Nrf2 antioxidant defense pathway probably via activating p38 signaling pathway. The activation of Nrf2 and inhibition of p38 significantly inhibited ox-LDL induced ROS production, mitochondria membrane potential reduction and cell apoptosis, and activated apoptotic pathway Bax /Bcl-2.

\section{Abbreviations}

ox-LDL: Oxidized-low density lipoprotein

ARE : Antioxidant response element

Nrf2: Nuclear factor erythroid 2- related factor 2

Keap1: Kelch-like ECH-associated protein-1

EPCs : Endothelial progenitor cells

MNCs: Mononuclear cells

PBS: Phosphate buffer saline

SDS-PAGE: Sodium dodecyl sulfate polyacrylamide gel electrophoresis

GAPDH: Glyceraldehyde-3-phosphate dehydrogenase

NQ01: NAD(P)H: quinone oxidoreductase 1 
H2DCF-DA: 2',7'-Dichlorofluorescin diacetate

ROS: Reactive oxygen species

MMP: Mitochondrial membrane potential

JC-1: 5,5',6,6'-Tetrachloro-1,1',3,3'-tetraethyl-imidacarbocyanine iodide

FITC: Fluorescein isothiocyanate

Pl: Propidium lodide

DAPI: 4',6-diamidino-2-phenylindole

FBS: Fetal bovine serum

t-Nrf2: total Nuclear factor erythroid 2- related factor 2

c-Nrf2: cytoplasmic Nuclear factor erythroid 2- related factor 2

n- Nrf2: nuclear Nuclear factor erythroid 2- related factor 2

MAPK: Mitogen-activated protein kinase

DHE: Dihydroethidium

$\mathrm{NAD}(\mathrm{P}) \mathrm{H}$ : Nicotinamide adenine dinucleotide (phosphate)

\section{Declarations}

Ethics approval and consent to participate

The informed consent for clinic and research usage of the blood sample were signed by blood donor. Red blood cells and plasma were extracted from the sample for clinic usage. Peripheral Blood Mononuclear Cells were obtained from the remaining samples for the preparation of EPCs. The study was approved by the Institutional Review Board at General Hospital of Central Theater Command.

Consent for publication

Not applicable.

Availability of data and materials

no

Competing interests 
The authors declare that they have no competing interests.

Funding

This work was supported by the National Science Foundation of China for Youth Scientist (81600318).

Authors' contributions

The study was designed by Qijun Jiang and Shifang Ding. Qijun Jiang carried out the experiments, performed the statistical analyses, and prepared the manuscript. Chengpeng Li囚Zhigang Gong, Zhigang $\mathrm{Li}$ contributed to some of the experiments and the statistical analyses. All authors have read and approved the final manuscript.

Acknowledgements

Not applicable.

\section{References}

1 Narverud I, RetterstøI K, Iversen PO, Halvorsen B, Ueland T, Ulven SM, Ose L, Aukrust P, Veierød MB, Holven KB. Markers of atherosclerotic development in children with familial hypercholesterolemia: a literature review. Atherosclerosis. 2014 Aug; 235(2):299-309.

2 Förstermann U, Xia N, Li H. Roles of Vascular Oxidative Stress and Nitric Oxide in the Pathogenesis of Atherosclerosis. Circ Res. 2017 Feb 17;120(4):713-735.

3 Haybar H, Shahrabi S, Rezaeeyan H, Shirzad R, Saki N. Endothelial Cells: From Dysfunction Mechanism to Pharmacological Effect in Cardiovascular Disease. Cardiovasc Toxicol. 2019 Feb;19(1):13-22.

4 Hong SH, Jang HH, Lee SR, Lee KH, Woo JS, Kim JB, Kim WS, Min BI, Cho KH, Kim KS, Cheng X, Kim W. Impact of lysophosphatidylcholine on survival and function of UEA-1(+)acLDL (+) endothelial progenitor cells in patients with coronary artery disease. Heart Vessels. 2015 Jan;30(1):115-25.

5 L Suraweera T, Rupasinghe HPV, Dellaire G, Xu Z. Regulation of Nrf2/ARE Pathway by Dietary Flavonoids: A Friend or Foe for Cancer Management? Antioxidants (Basel). 2020 Oct 11;9(10):973.

6 da Costa RM, Rodrigues D, Pereira CA, Silva JF, Alves JV, Lobato NS, Tostes RC. Nrf2 as a Potential Mediator of Cardiovascular Risk in Metabolic Diseases. Front Pharmacol. 2019 Apr 12;10:382.

7 Chen QM, Maltagliati AJ. Nrf2 at the heart of oxidative stress and cardiac protection. Physiol Genomics. 2018 Feb 1;50(2):77-97.

8 Liu Q, Gao Y, Ci X. Role of Nrf2 and Its Activators in Respiratory Diseases. Oxid Med Cell Longev. 2019 Jan 8;2019:7090534. 
9 Bhattacharjee S, Dashwood RH. Epigenetic Regulation of NRF2/KEAP1 by Phytochemicals. Antioxidants (Basel). 2020 Sep 14;9(9):865.

10 Ishii T, Itoh K, Ruiz E, Leake DS, Unoki H, Yamamoto M, Mann GE. Role of Nrf2 in the regulation of CD36 and stress protein expression in murine macrophages: activation by oxidatively modified LDL and 4-hydroxynonenal. Circ Res. 2004 Mar 19;94(5):609-16.

10 Furman C, Rundlöf AK, Larigauderie G, Jaye M, Bricca G, Copin C, Kandoussi AM, Fruchart JC, Arnér ES, Rouis M. Thioredoxin reductase 1 is upregulated in atherosclerotic plaques: specific induction of the promoter in human macrophages by oxidized low-density lipoproteins. Free Radic Biol Med. $2004 \mathrm{Jul}$ 1;37(1):71-85.

11 Jiang Q, Ding S, Wu J, Liu X, Wu Z. Norepinephrine stimulates mobilization of endothelial progenitor cells after limb ischemia. PLoS One. 2014 Jul 9;9(7):e101774.

12 Bao XM, Wu CF, Lu GP. Atorvastatin inhibits homocysteine-induced oxidative stress and apoptosis in endothelial progenitor cells involving Nox4 and p38MAPK. Atherosclerosis. 2010 May;210(1):114-21.

13 Wang R, Liu L, Liu H, Wu K, Liu Y, Bai L, Wang Q, Qi B, Qi B, Zhang L. Reduced NRF2 expression suppresses endothelial progenitor cell function and induces senescence during aging. Aging (Albany NY). 2019 Sep 8;11(17):7021-7035.

14 Tang Y, Jacobi A, Vater C, Zou X, Stiehler M. Salvianolic acid B protects human endothelial progenitor cells against oxidative stress-mediated dysfunction by modulating Akt/mTOR/4EBP1, p38 MAPK/ATF2, and ERK1/2 signaling pathways. Biochem Pharmacol. 2014 Jul 1;90(1):34-49.

15 Diniyah N, Alam MB, Choi HJ, Lee SH. Lablab Purpureus Protects HaCaT Cells from Oxidative StressInduced Cell Death through Nrf2-Mediated Heme Oxygenase-1 Expression via the Activation of p38 and ERK1/2. Int J Mol Sci. 2020 Nov 14;21(22):8583.

16 Zeng YC, Peng LS, Zou L, Huang SF, Xie Y, Mu GP, Zeng XH, Zhou XL, Zeng YC. Protective effect and mechanism of lycopene on endothelial progenitor cells (EPCs) from type 2 diabetes mellitus rats. Biomed Pharmacother. 2017 Aug;92:86-94.

17 Papachristoforou E, Lambadiari V, Maratou E, Makrilakis K. Association of Glycemic Indices (Hyperglycemia, Glucose Variability, and Hypoglycemia) with Oxidative Stress and Diabetic Complications. J Diabetes Res. 2020 Oct 12;2020:7489795.

18 Vancheri F, Longo G, Vancheri S, Henein M. Coronary Microvascular Dysfunction. J Clin Med. 2020 Sep 6;9(9):2880.

19 Balaji S, King A, Crombleholme TM, Keswani SG. The Role of Endothelial Progenitor Cells in Postnatal Vasculogenesis: Implications for Therapeutic Neovascularization and Wound Healing. Adv Wound Care (New Rochelle). 2013 Jul;2(6):283-295. 
20 Lai P, Liu Y. Echinocystic acid, isolated from Gleditsia sinensis fruit, protects endothelial progenitor cells from damage caused by oxLDL via the Akt/eNOS pathway. Life Sci. 2014 Oct 2;114(2):62-9.

21 Wang J, Chen S, Ma X, Cheng C, Xiao X, Chen J, Liu S, Zhao B, Chen Y. Effects of endothelial progenitor cell-derived microvesicles on hypoxia/reoxygenation-induced endothelial dysfunction and apoptosis. Oxid Med Cell Longev. 2013;2013:572729.

22 Wu Y, Song F, Li Y, Li J, Cui Y, Hong Y, Han W, Wu W, Lakhani I, Li G, Wang Y. Acacetin exerts antioxidant potential against atherosclerosis through Nrf2 pathway in apoE-/- Mice. J Cell Mol Med. 2020 Nov 26. doi: $10.1111 /$ jcmm.16106. Epub ahead of print. PMID: 33241629.

23 Jin Z, Xiao Y, Yao F, Wang B, Zheng Z, Gao H, Lv X, Chen L, He Y, Wang W, Lin R. SIRT6 inhibits cholesterol crystal-induced vascular endothelial dysfunction via Nrf2 activation. Exp Cell Res. 2020 Feb 1;387(1):111744.

24 Saha S, Buttari B, Panieri E, Profumo E, Saso L. An Overview of Nrf2 Signaling Pathway and Its Role in Inflammation. Molecules. 2020 Nov 23;25(22):5474.

25 Li S, Shi M, Wang Y, Xiao Y, Cai D, Xiao F. Keap1-Nrf2 pathway up-regulation via hydrogen sulfide mitigates polystyrene microplastics induced-hepatotoxic effects. J Hazard Mater. 2021 Jan 15;402:123933.

26 Ahn YJ, Lim JW, Kim H. Docosahexaenoic Acid Induces Expression of NAD(P)H: Quinone Oxidoreductase and Heme Oxygenase-1 through Activation of Nrf2 in Cerulein-Stimulated Pancreatic Acinar Cells. Antioxidants (Basel). 2020 Nov 4;9(11):1084.

27 Li W, Yu J, Xiao X, Zang L, Yang Y, Yu J, Huang Q, Niu X, Li W. Imperatorin reduces the inflammatory response of atherosclerosis by regulating MAPKs signaling pathway in vivo and in vitro. Int Immunopharmacol. 2020 Nov 17:107170.

28 Zhao J, Peng W, Ran Y, Ge H, Zhang C, Zou H, Ding Y, Qi H. Dysregulated expression of ACTN4 contributes to endothelial cell injury via the activation of the p38-MAPK/p53 apoptosis pathway in preeclampsia. J Physiol Biochem. 2019 Nov;75(4):475-487.

29 Gong L, Lei Y, Liu Y, Tan F, Li S, Wang X, Xu M, Cai W, Du B, Xu F, Zhou Y, Han H, Sun H, Qiu L. Vaccarin prevents ox-LDL-induced HUVEC EndMT, inflammation and apoptosis by suppressing ROS/p38 MAPK signaling. Am J TransI Res. 2019 Apr 15;11(4):2140-2154.

30 Fão L, Mota SI, Rego AC. Shaping the Nrf2-ARE-related pathways in Alzheimer's and Parkinson's diseases. Ageing Res Rev. 2019 Sep;54:100942.

31 Chen H, Strappe P, Chen S, Wang LX. Endothelial progenitor cells and pulmonary arterial hypertension. Heart Lung Circ. 2014 Jul;23(7):595-601. 
32 Mudyanadzo TA. Endothelial Progenitor Cells and Cardiovascular Correlates. Cureus. 2018 Sep 21;10(9):e3342.

33 Zhao R, Feng J, He G. Hypoxia increases Nrf2-induced HO-1 expression via the PI3K/Akt pathway. Front Biosci (Landmark Ed). 2016 Jan 1;21:385-96.

34 Vitagliano O, Addeo R, D'Angelo V, Indolfı C, Indolfı P, Casale F. The Bcl-2/Bax and Ras/Raf/MEK/ERK signaling pathways: implications in pediatric leukemia pathogenesis and new prospects for therapeutic approaches. Expert Rev Hematol. 2013 Oct;6(5):587-97.

35 D'Orsi B, Mateyka J, Prehn JHM. Control of mitochondrial physiology and cell death by the Bcl-2 family proteins Bax and Bok. Neurochem Int. 2017 Oct;109:162-170.

\section{Figures}
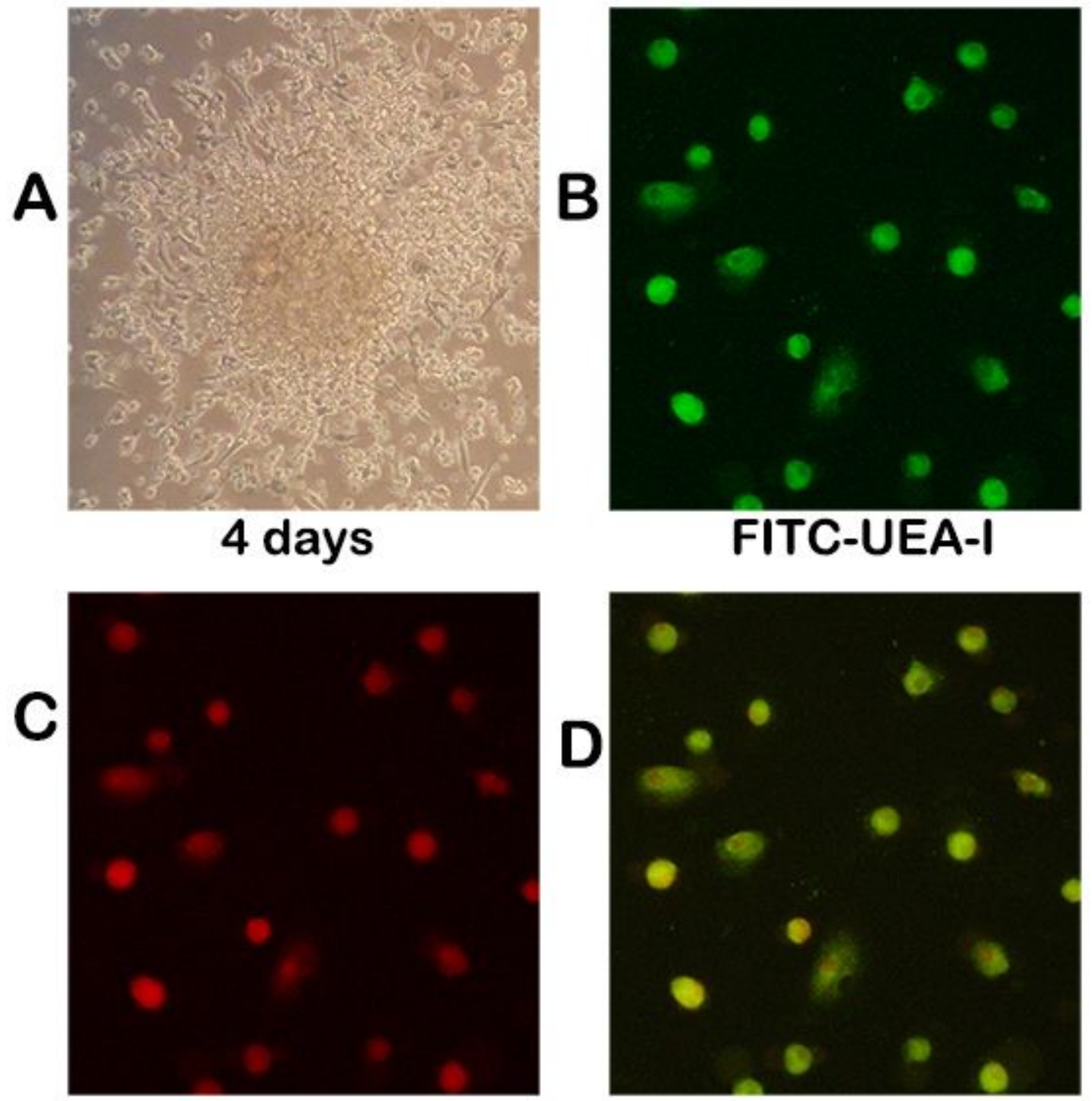

Dil-ac-LDL

Merged 
Figure 1

A caption was not provided with this version

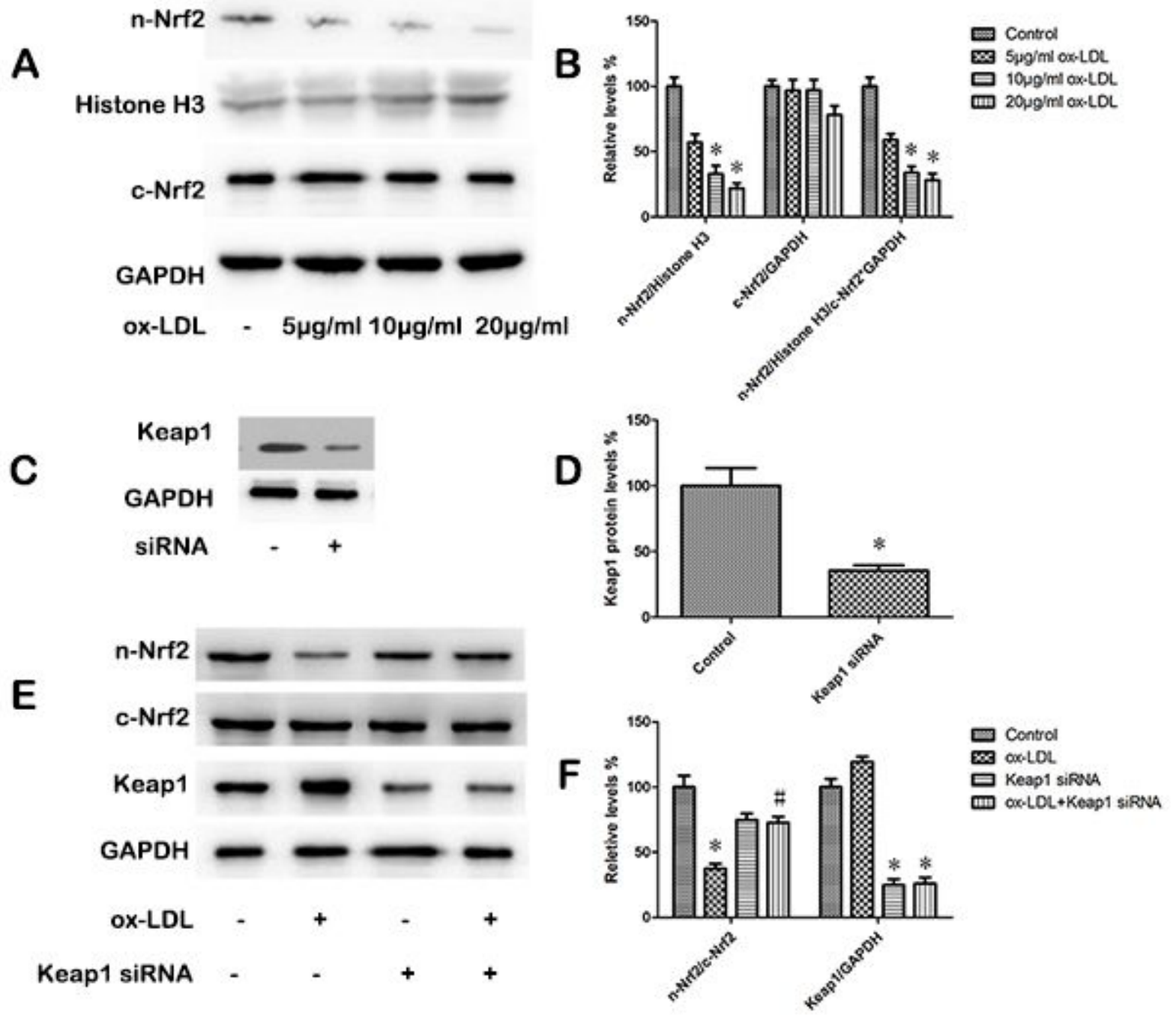

Figure 2

A caption was not provided with this version 


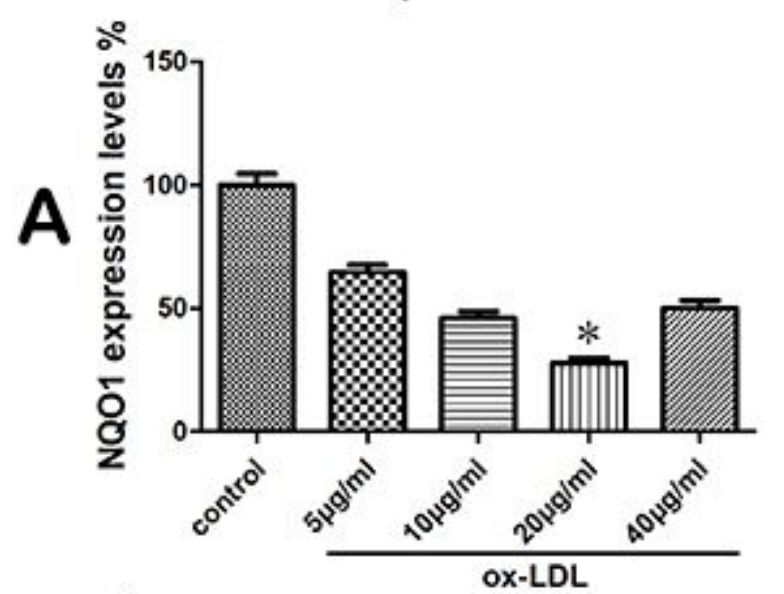

c
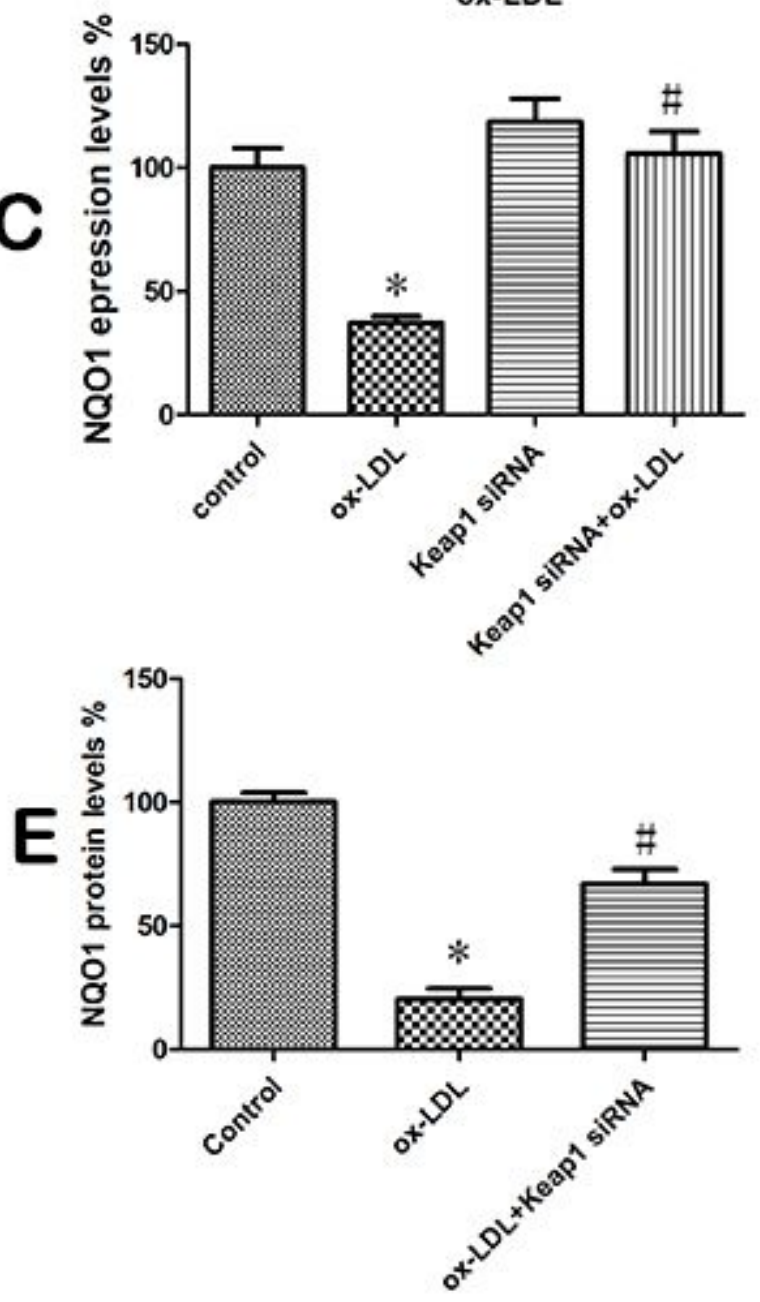

Figure 3

A caption was not provided with this version
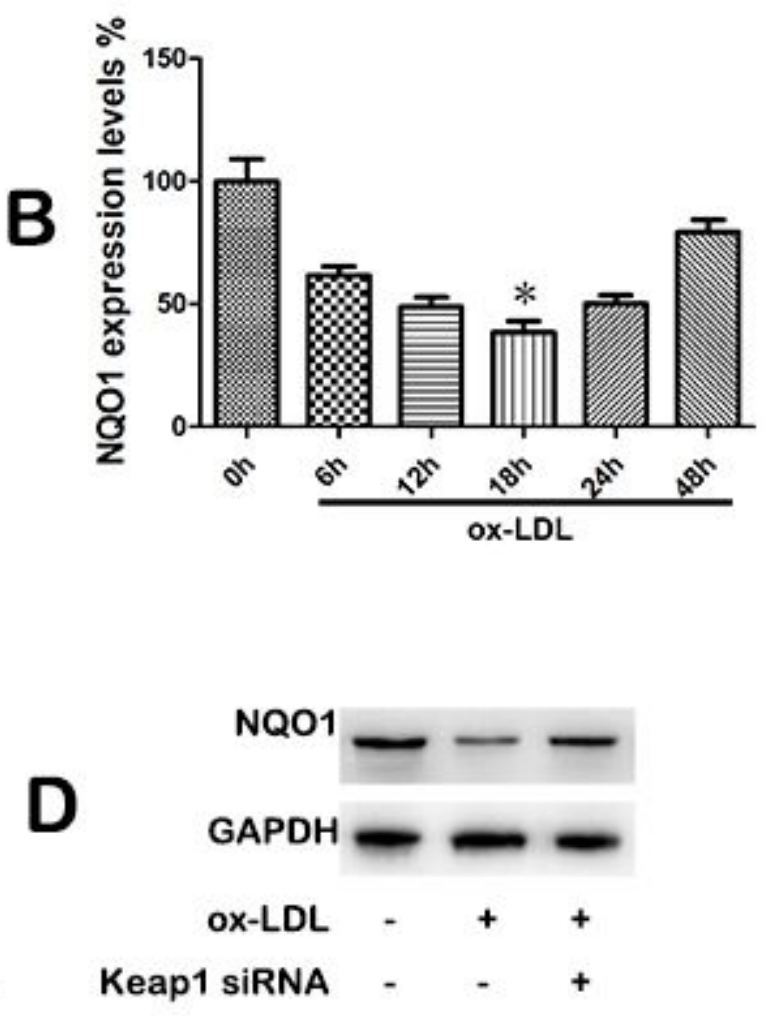


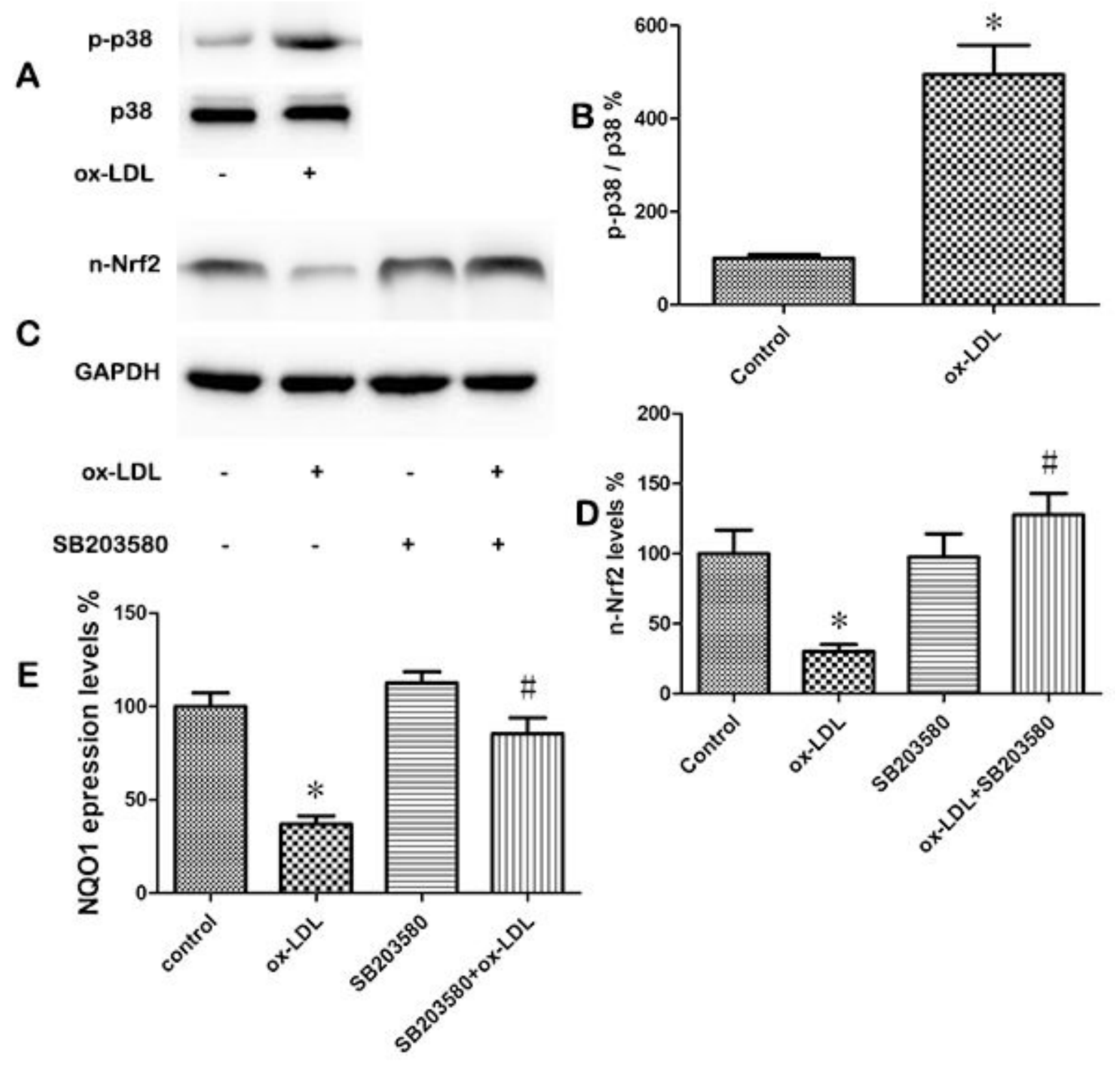

Figure 4

A caption was not provided with this version 


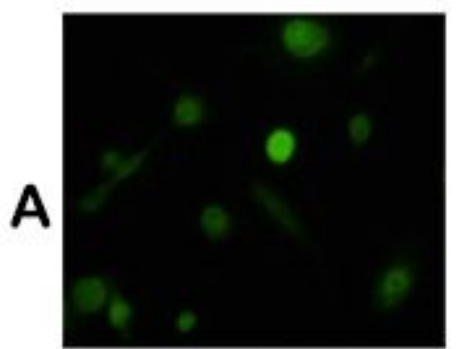

Control

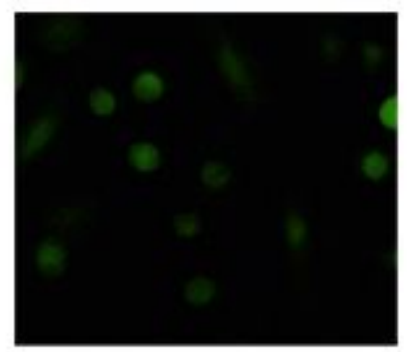

ox-LDL+Keap1 siRNA

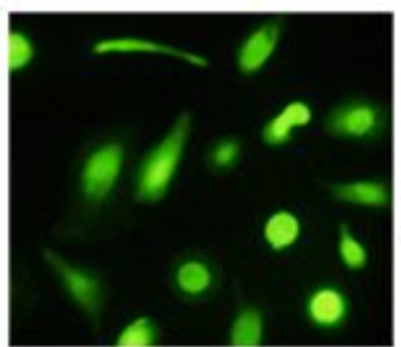

ox-LDL

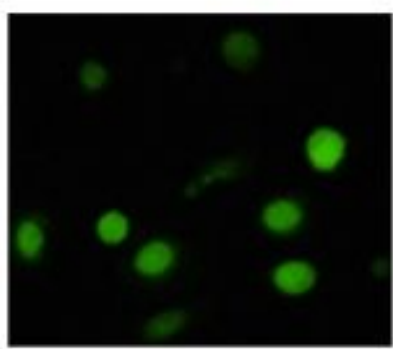

ox-LDL+SB203580

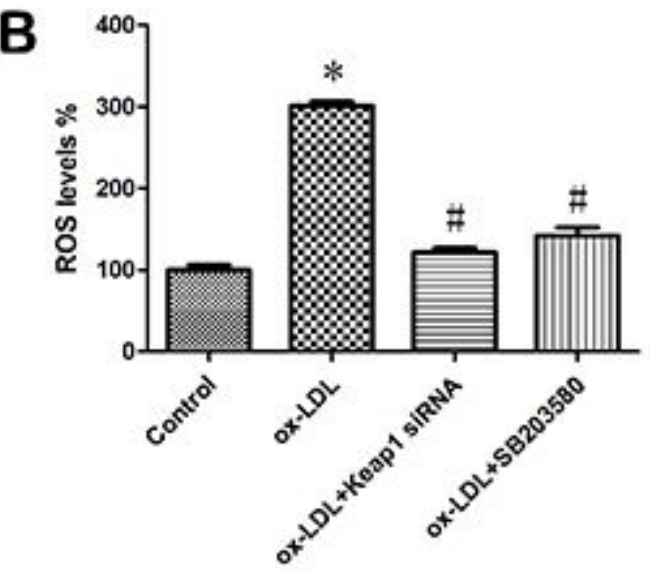

Figure 5

A caption was not provided with this version

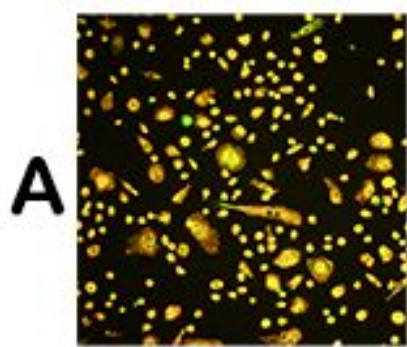

Control

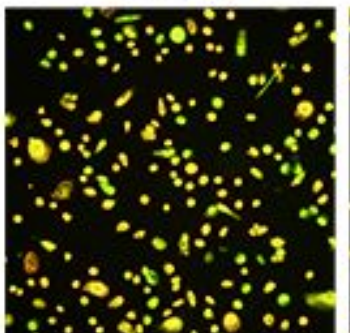

ox-LDL+Keap1 siRNA ox-LDL+SB203580

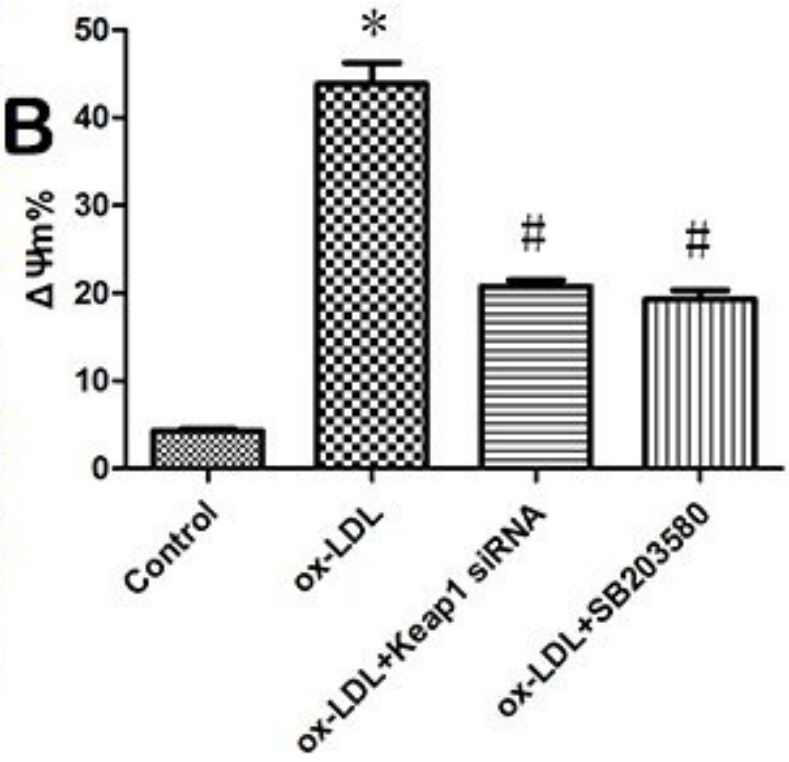

Figure 6

A caption was not provided with this version 

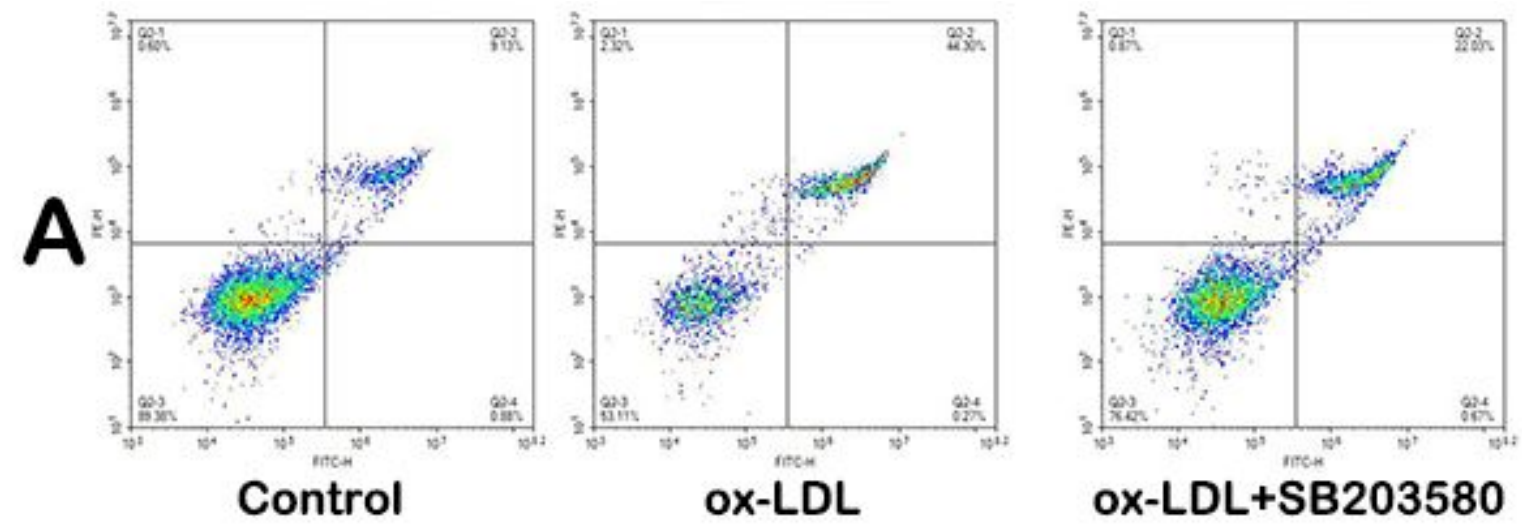

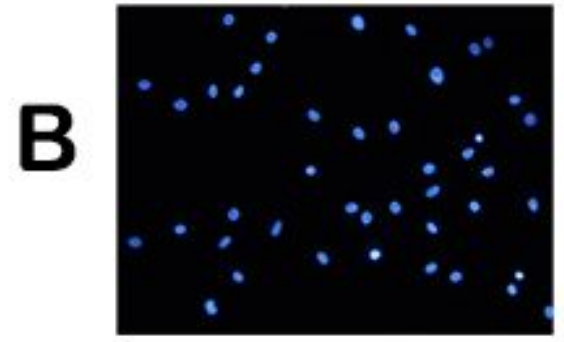

Control

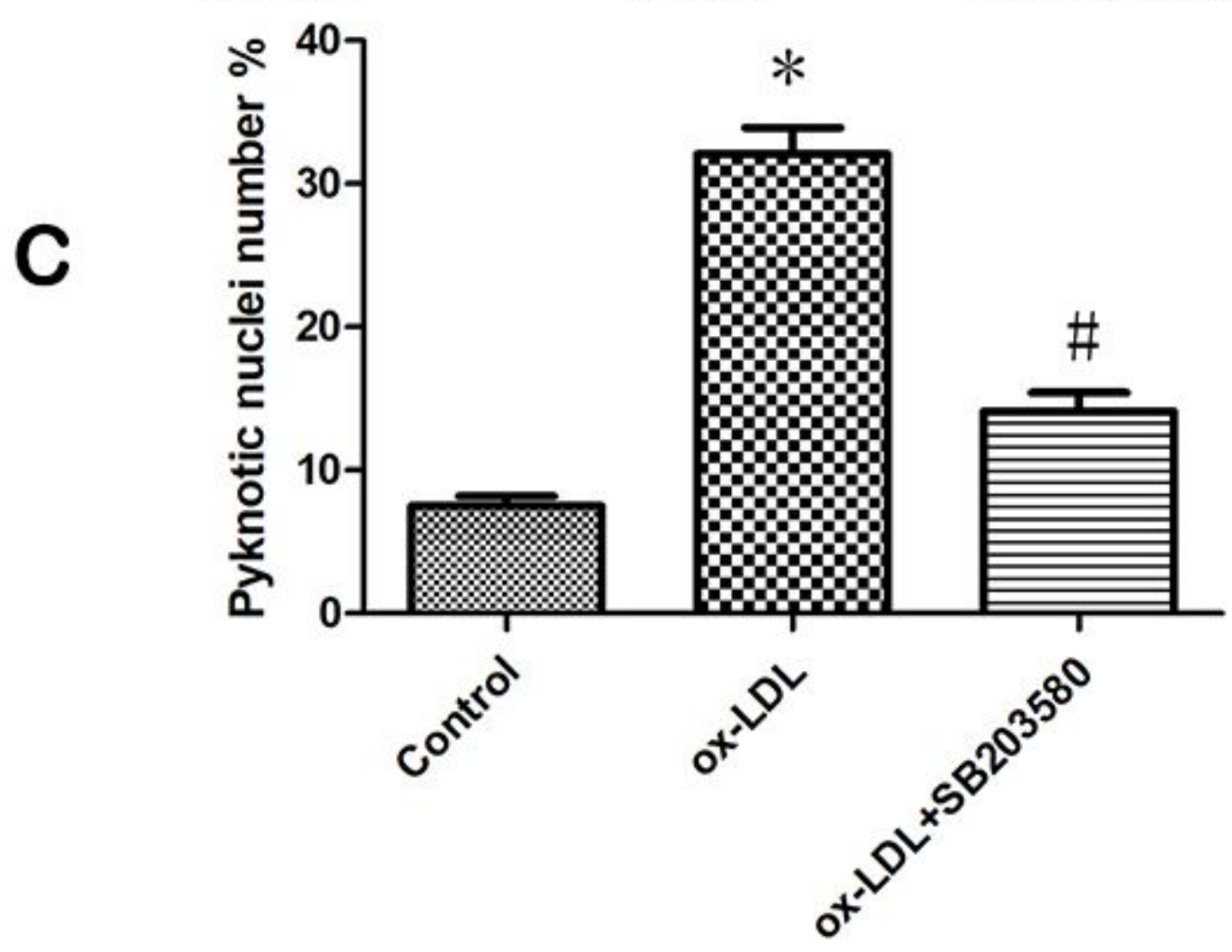

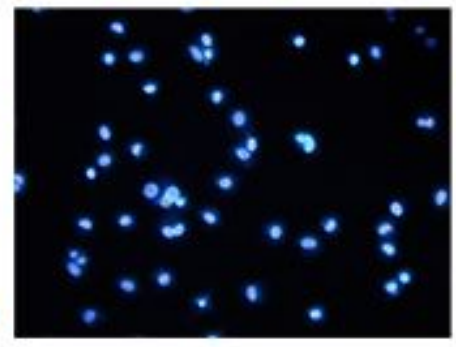

ox-LDL

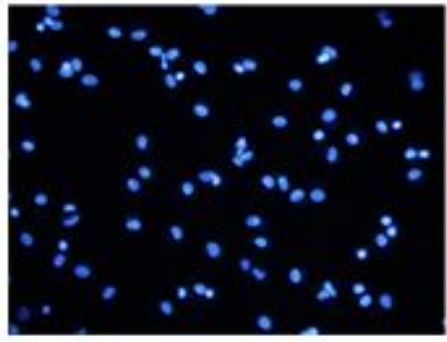

ox-LDL+SB203580

Figure 7

A caption was not provided with this version 


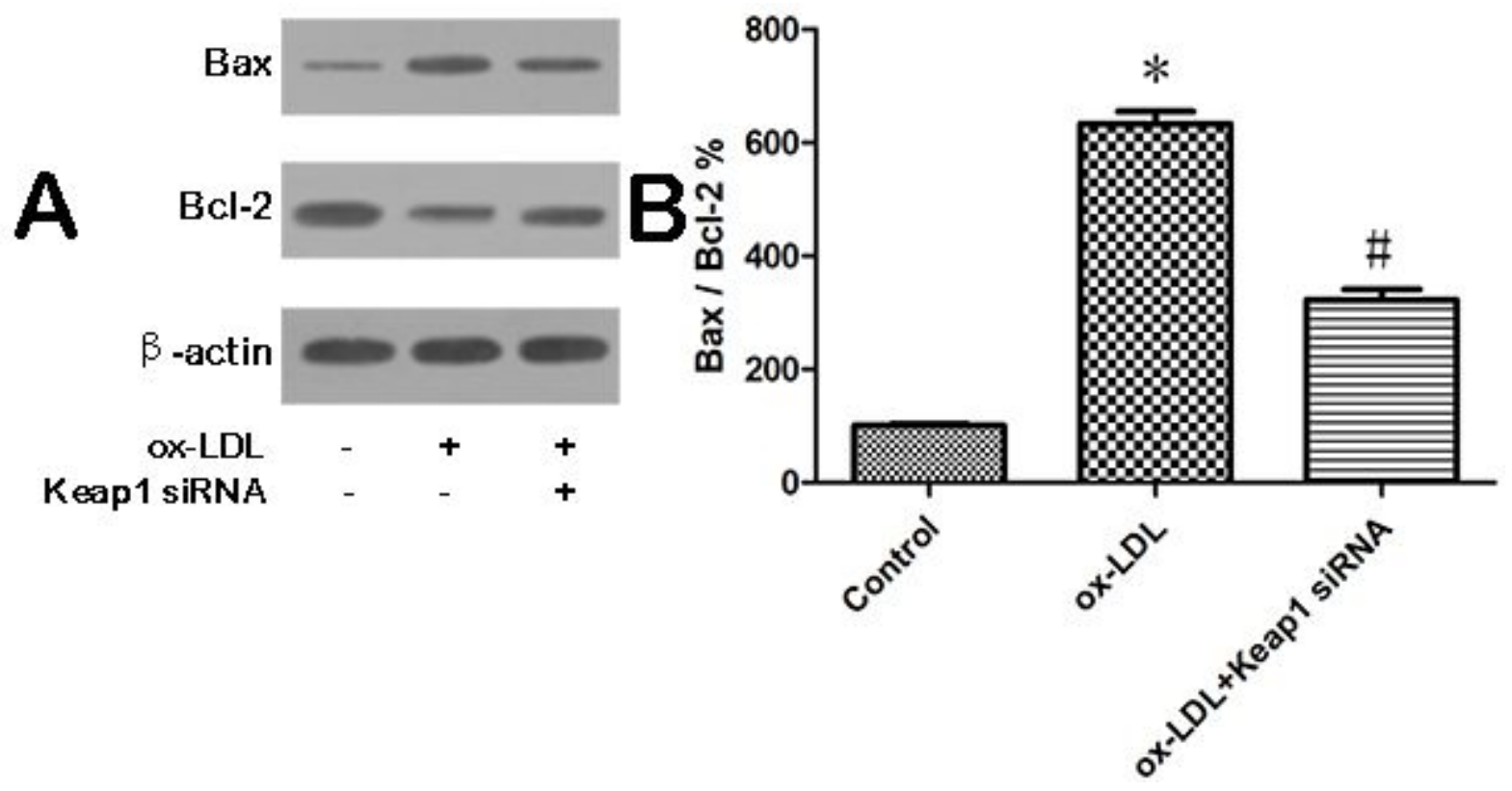

Figure 8

A caption was not provided with this version

A

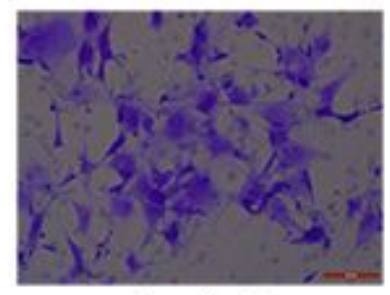

Control

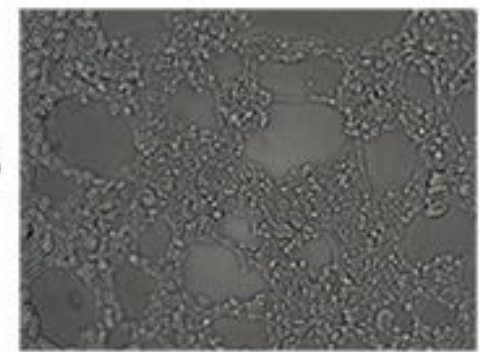

Control

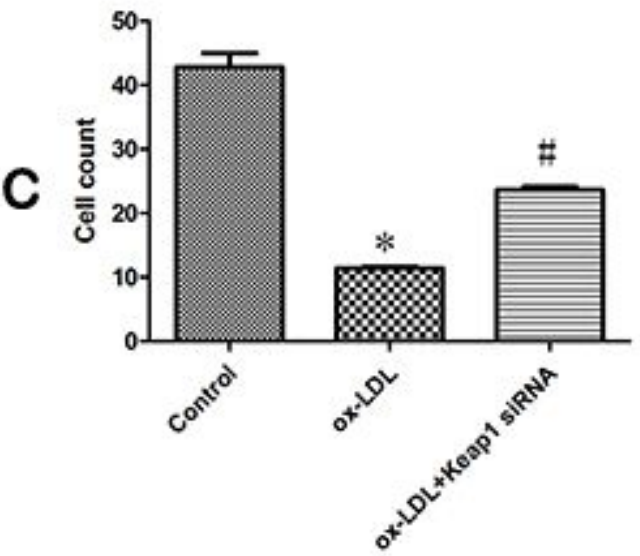

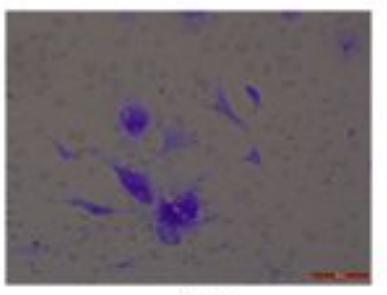

ox-LDL

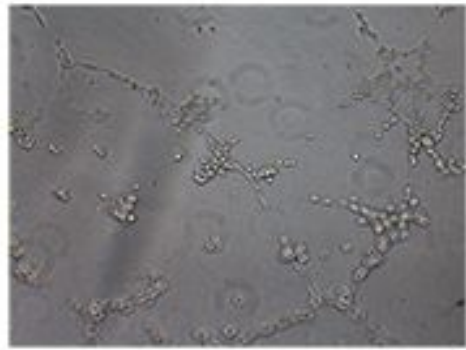

ox-LDL

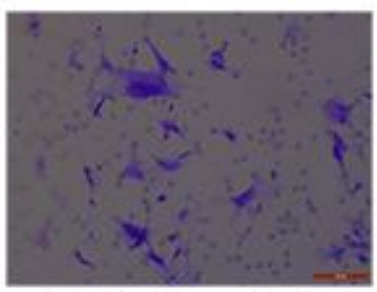

ox-LDL+Keap1 siRNA

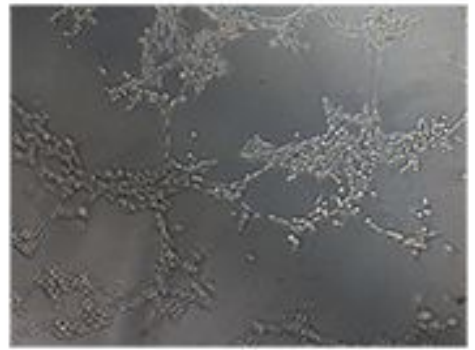

ox-LDL+Keap1 siRNA

D

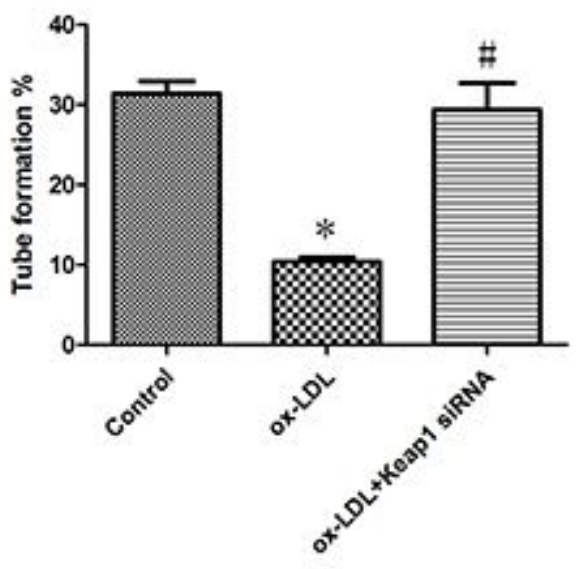

Figure 9 
A caption was not provided with this version

Page 23/23 